\title{
Effect of serrated trailing edges on aerofoil tonal noise
}

\author{
Matthieu B. R. Gelot ${ }^{1} \dagger$, and Jae Wook Kim ${ }^{1}$ \\ ${ }^{1}$ Institute of Sound and Vibration Research, University of Southampton, Highfield, \\ Southampton, SO17 1BJ, UK
}

A wall-resolved large-eddy simulation of a symmetric Joukowski aerofoil with a $12 \%$ thickness at $R e_{\infty}=250000, M_{\infty}=0.4$ and zero incidence angle is performed in order to investigate the effect of using a serrated trailing edge on the acoustic feedback event that generates a tonal noise. The acoustic feedback is investigated in details to emphasise the interaction between the upstream travelling acoustic expansion wave and the laminar separation bubble. The simulation shows that the serrated trailing edges may result in a significant reduction of the tonal noise. This paper provides detailed investigations into the noise reduction mechanisms. The main finding is that the presence of a serrated trailing edge decreases the amplitude of the acoustic source pressure in the transitional region and gives rise to destructive phase interference in the wall pressure fluctuations in the vicinity of the trailing edge which weakens the acoustic feedback loop.

\section{Introduction}

It is well known that aerofoil noise can be split into several different types (Brooks et al. 1989). Among the various natures of aerofoil noise, Fink (1975) investigated the tonal noise generated due to laminar-to-turbulent boundary-layer transition. They showed the existence of a law predicting the tone frequencies from the boundary-layer instability theory. Meanwhile, Paterson et al. (1973) observed that the tone frequencies scaled proportionally to the $3 / 2$ power of the free-stream velocity. A feedback loop theory was introduced by Tam (1974). They inferred that the feedback loop took place between the aerofoil trailing edge and a location in the wake. However, this theory was challenged by Longhouse (1977) who suggested that the feedback loop occurred between the acoustic waves radiating from the trailing edge and the Tollmien-Schlichting (T-S) instability waves developing in the transition region. Arbey \& Bataille (1983) confirmed Paterson's work and suggested that the tones were predicted using the following formula:

$$
\frac{f_{n} L_{c}}{a_{\infty}}=\frac{U_{c} / a_{\infty}}{L / L_{c}}\left(n+\frac{1}{2}\right)\left(1+\frac{U_{c} / a_{\infty}}{1-M_{\infty}}\right)^{-1}
$$

where $a_{\infty}$ is the speed of sound, $L_{c}$ is the aerofoil chord length, $U_{c} / a_{\infty}$ is the nondimensional convection speed of the boundary-layer, $M_{\infty}=U_{\infty} / a_{\infty}$ is the free-stream Mach number, $L / L_{c}$ is the distance separating the feedback locations and $n$ is an integer. The equation which is a modified version of the earlier one introduced by Tam (1974) clarifies that the feedback loop takes place between the trailing edge and the transitional region. Acoustic pressure waves scattered by the trailing edge propagate upstream at the speed of sound and trigger T-S instabilities in the transition region which will further generate hydrodynamic disturbances convecting downstream within the boundary-layer at convection speed $U_{c}$. The incoming hydrodynamic waves are then radiated by the

$\dagger$ Email address for correspondence: m.b.r.gelot@soton.ac.uk 
trailing edge and the process reiterates at a discrete frequency. This fixed frequency depends on the speed of sound, the convection speed and the distance separating the transitional region from the trailing edge.

Nash et al. (1999) and McAlpine et al. (1999) discussed the modelling of tonal noise in the case of a separating laminar boundary-layer in the vicinity of the trailing edge. Their results were further confirmed by Tam \& Ju (2012) in a direct numerical simulation. After many experimental investigations, Desquesnes et al. (2007) performed a direct numerical simulation to study tonal noise mechanisms and inferred that secondary discrete frequencies are created due to a bifurcation of the aerofoil wake from a symmetric to an asymmetric vortex pattern. Jones \& Sandberg (2011) showed that the tonal contribution becomes less significant compared to the broadband noise at high angles of attack. Arcondoulis et al. (2013) used formula (1.1) and found a satisfying agreement between the theory and their experimental observations. Chong et al. (2013) experimentally studied the tonal noise of a NACA0012 aerofoil for various angles of attack and Reynolds numbers. They found that the most effective tonal noise requires the T-S waves to be amplified by a separated boundary-layer near the trailing edge. This was further supported by Pröbsting et al. (2014) and Pröbsting et al. (2015) from their Particle Image Velocimetry (PIV) measurements in the same range of Reynolds numbers and angles of attack. Nguyen et al. (2017) distinguished the vortex shedding frequency from the acoustic feedback loop frequency.

An additional study on controlled-diffusion (CD) aerofoil was investigated by Padois et al. (2016) and Sanjose et al. (2017). The latter emphasised quiet and intense time strands where the quiet period is driven by attached boundary-layers and the intense period involves an unstable separation bubble. To summarise, coherent T-S instabilities coupled with the presence of a recirculation bubble in the vicinity of the trailing edge is the most effective condition for generating an aerofoil tonal noise.

An efficient method to decrease the aerofoil tonal noise would be of interest to many. The authors, however, have not identified various methods in this regard except the modified TE studied by Chong \& Joseph (2013). They tested four different sawtooth serration geometries fitted to a NACA0012 aerofoil. They did observe a reduction of the tonal noise when the serrated TEs were used, compared to an unmodified TE. They suggested that the reduction might be related to an abatement of the two-dimensionality in the T-S waves near the TE leading to a decorrelated T-S amplification. They also hypothesised that the serrated TE would be more effective if the separation bubble is located closer to the TE.

Following the work of Chong \& Joseph (2013), the main objective of this paper is to clarify the hypothesis of the tonal noise reduction due to the serrated TE. For this purpose, the authors use a high-fidelity numerical simulation to provide detailed flow and acoustic information. The noise reduction mechanisms found in this paper are twofold: 1) the presence of serrated TE diminishes the amplitude of the acoustic source pressure in the transitional region which lowers the acoustic source at the TE and 2) there is an elevated level of destructive phase interference in the acoustic source when a serrated TE is used.

\section{Computational details}

This section aims to introduce the governing equations, numerical methods and computational set-up of the present study. The current numerical investigation utilises a high-resolution implicit large-eddy simulation (LES) approach based on a wavenumberoptimised discrete filter. The filter acts as an implicit subgrid scale (SGS) model that 
dissipates scales smaller than the filter cut-off. It has been demonstrated by Garmann et al. (2013) and many others that an implicit LES is capable of capturing flow physics as accurately as the conventional SGS model based LES.

\subsection{Governing equations and numerical schemes}

The present numerical study is based on a wall-resolved large-eddy simulation (LES) by using the three-dimensional compressible Navier-Stokes equations. The Navier-Stokes equations (with a source term for the sponge layer implementation) can be written in a conservative form, transformed onto a generalised coordinate system as

$$
\frac{\partial}{\partial t}\left(\frac{\boldsymbol{Q}}{J}\right)+\frac{\partial}{\partial \xi_{i}}\left(\frac{\boldsymbol{E}_{j}-R e_{\infty}^{-1} M_{\infty} \boldsymbol{F}_{j}}{J} \frac{\partial \xi_{i}}{\partial x_{j}}\right)=-\frac{a_{\infty}}{L_{c}} \frac{\boldsymbol{S}}{J},
$$

where the indices $i=1,2,3$ and $j=1,2,3$ denote the three dimensions; and, $a_{\infty}$ is the ambient speed of sound. The vectors of the conservative variables, inviscid and viscous fluxes are given by

$$
\left.\begin{array}{c}
\boldsymbol{Q}=\left[\rho, \rho u, \rho v, \rho w, \rho e_{\mathrm{t}}\right]^{T}, \\
\boldsymbol{E}_{j}=\left[\rho u_{j},\left(\rho u u_{j}+\delta_{1 j} p\right),\left(\rho v u_{j}+\delta_{2 j} p\right),\left(\rho w u_{j}+\delta_{3 j} p\right),\left(\rho e_{\mathrm{t}}+p\right) u_{j}\right]^{T}, \\
\boldsymbol{F}_{j}=\left[0, \tau_{1 j}, \tau_{2 j}, \tau_{3 j}, u_{i} \tau_{j i}+q_{j}\right]^{T},
\end{array}\right\}
$$

with the stress tensor and heat flux vector written as

$$
\tau_{i j}=\mu\left(\frac{\partial u_{i}}{\partial x_{j}}+\frac{\partial u_{j}}{\partial x_{i}}-\frac{2}{3} \delta_{i j} \frac{\partial u_{i}}{\partial x_{i}}\right), \quad q_{j}=\frac{\mu}{(\gamma-1) \operatorname{Pr}} \frac{\partial T}{\partial x_{j}},
$$

where $\xi_{i}=\{\xi, \eta, \zeta\}$ are the generalised coordinates, $x_{j}=\{x, y, z\}$ are the Cartesian coordinates, $\delta_{i j}$ is the Kronecker delta, $u_{j}=\{u, v, w\}, e_{\mathrm{t}}=p /[(\gamma-1) \rho]+u_{j} u_{j} / 2$ and $\gamma=1.4$ for air. The local dynamic viscosity $\mu$ is calculated by using Sutherland's law (White 1991). In the current set-up, $\xi, \eta$ and $\zeta$ are aligned in the streamwise, wallnormal and spanwise directions, respectively. The Jacobian determinant of the coordinate transformation (from Cartesian to generalised) is given by $J^{-1}=|\partial(x, y, z) / \partial(\xi, \eta, \zeta)|$. In this paper, the free-stream Mach and Reynolds numbers are defined as $M_{\infty}=u_{\infty} / a_{\infty}$ and $R e_{\infty}=\rho_{\infty} u_{\infty} L_{c} / \mu_{\infty}$. The Mach number is fixed at $M_{\infty}=0.4$ and a Reynolds number of $R e_{\infty}=2.5 \times 10^{5}$ is considered. The governing equations are non-dimensionalised based on the aerofoil chord length $L_{c}$ for length scales, the ambient speed of sound $a_{\infty}$ for velocities, $L_{c} / a_{\infty}$ for time scales and $\rho_{\infty} a_{\infty}^{2}$ for pressure. Temperature, density and dynamic viscosity are normalised by their respective ambient values: $\Theta_{\infty}, \rho_{\infty}$ and $\mu_{\infty}$.

The spatial derivatives are calculated by using an optimised pentadiagonal compact finite difference scheme that is globally fourth-order accurate up to the boundaries (Kim 2007). A matching fourth-order Runge-Kutta scheme is used for explicit time marching with a Courant-Friedrichs-Lewy (CFL) number of 1.0. A sixth-order pentadiagonal compact filter with a normalised cut-off wavenumber of $0.85 \pi$ (Kim 2010) is used to dissipate unresolved sub-grid scales and ensure the numerical stability. The computation is distributed over 512 processor cores based on a message passing interface (MPI) parallelisation. The MPI parallelisation of the compact scheme and filter is implemented by using the quasi-disjoint matrix system proposed by Kim (2013). All simulations are executed on IRIDIS-4 at the University of Southampton.

\subsection{Computational domain, grid and boundary conditions}

The present simulation is based on a symmetric Joukowski aerofoil with a $12 \%$ thickness. We consider three different trailing edge (TE) geometries in this study: baseline 

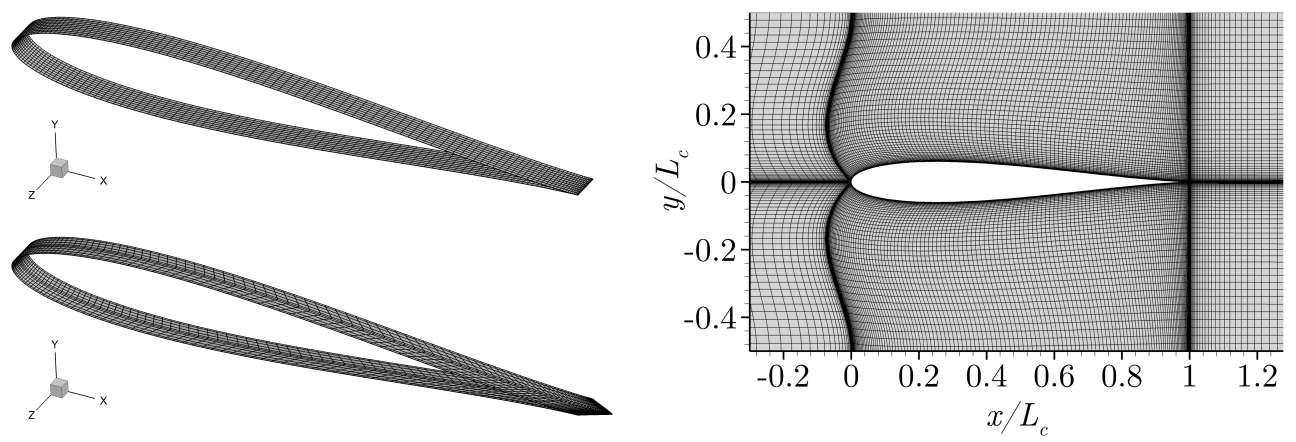

Figure 1. A bird's eye view of the surface mesh for the BTE and STE2 aerofoils (20\% of total cells shown) on the left and a cross-sectional view of the interior mesh of the BTE case (25\% of total cells shown) on the right.

(BTE) and two serrated (STE) cases. The conventional sawtooth serrations are used. Grid meshes used in the current work are displayed in figure 1. A structured H-type topology is used in the present grid and the cells are gradually stretched from the aerofoil surface to the far boundaries. In the BTE case, a constant spanwise grid spacing is used. Meanwhile, in the STE cases, a finer grid spacing is used near the sharp corners. The tips and roots of the serrations are gently rounded (with a radius of $3 \%$ of the span) in order to maintain a continuous mesh without losing the sharp character of the serration. The aerofoil is centred at the origin of the domain. In the BTE case, the leading and trailing edges lie at $x / L_{c}=0.0$ and 1.0, respectively. In the paper, two different STE geometries are considered; STE1 with an aspect ratio of 0.5 and STE2 of 1.0. The aspect ratio is defined by the streamwise length divided by the spanwise wavelength of the serration. The serrations extend from the baseline geometry. The local chord length is the same as the baseline case at the roots of the serrations and it becomes longer at the tips.

The spanwise domain size is set to $5 \%$ of the chord length $\left(L_{z}=0.05 L_{c}\right)$ of the baseline aerofoil. This span length is also set as the wavelength of the serrations. The aerofoil chord is aligned with the $x$-axis. The entire grid consists of $N_{\xi} \times N_{\eta} \times N_{\zeta}=961 \times 481 \times 51=23.6$ million points where $N_{\xi}, N_{\eta}$ and $N_{\zeta}$ are the streamwise, vertical and spanwise number of points. The wall-unit grid spacings are presented in figure 2 and they all meet the wallresolved LES criteria recommended by Georgiadis et al. (2010): $\Delta s^{+}<40, \Delta z^{+}<40$ and $\Delta n^{+}<1$ in the turbulent region where $\Delta s^{+}, \Delta n^{+}$and $\Delta z^{+}$are the body-fitted streamwise, wall-normal and spanwise wall-unit grid spacings, respectively.

Characteristics-based non-reflecting boundary conditions (Kim \& Lee 2000) are used in the far field boundaries. A sponge layer (Kim et al. 2010a,b) is introduced via the source term $\boldsymbol{S}$ in (2.1) around the far field boundaries to enforce the damping of acoustic waves and therefore avoid spurious reflections. A no-slip iso-thermal wall boundary condition is applied on the aerofoil surface. A periodic boundary condition is applied across the spanwise boundaries.

Each simulation is run for $t u_{\infty} / L_{c}=16.0$ time units in total where the fully developed solutions are taken during the last 4.0 time units for data processing. In figure 3, timeand spanwise-averaged profiles of the pressure and skin friction coefficients $\left(C_{p}\right.$ and $\left.C_{f}\right)$ are compared with corresponding XFoil predictions. It is shown that the time-averaged solution matches very well with the XFoil prediction over the laminar region of the flow. There is a noticeable deviation in the turbulent region perhaps attributed to the difference between the two- and three-dimensional approaches. 

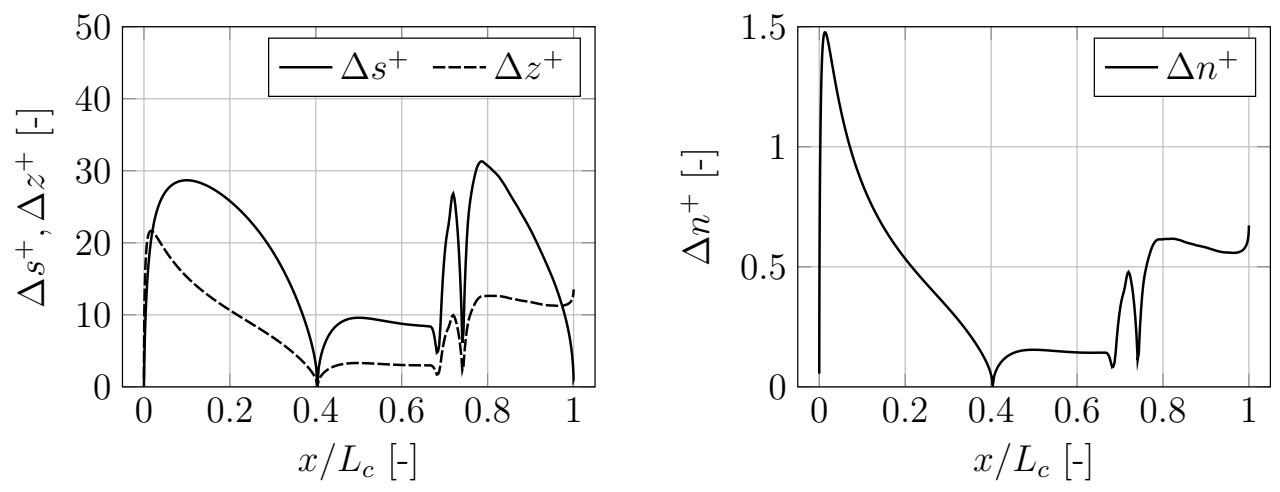

FIGURE 2. Wall-unit grid spacings used in the BTE case where $\Delta s^{+}$is the streamwise curvilinear spacing, $\Delta z^{+}$is the spanwise spacing and $\Delta n^{+}$is the wall-normal spacing.
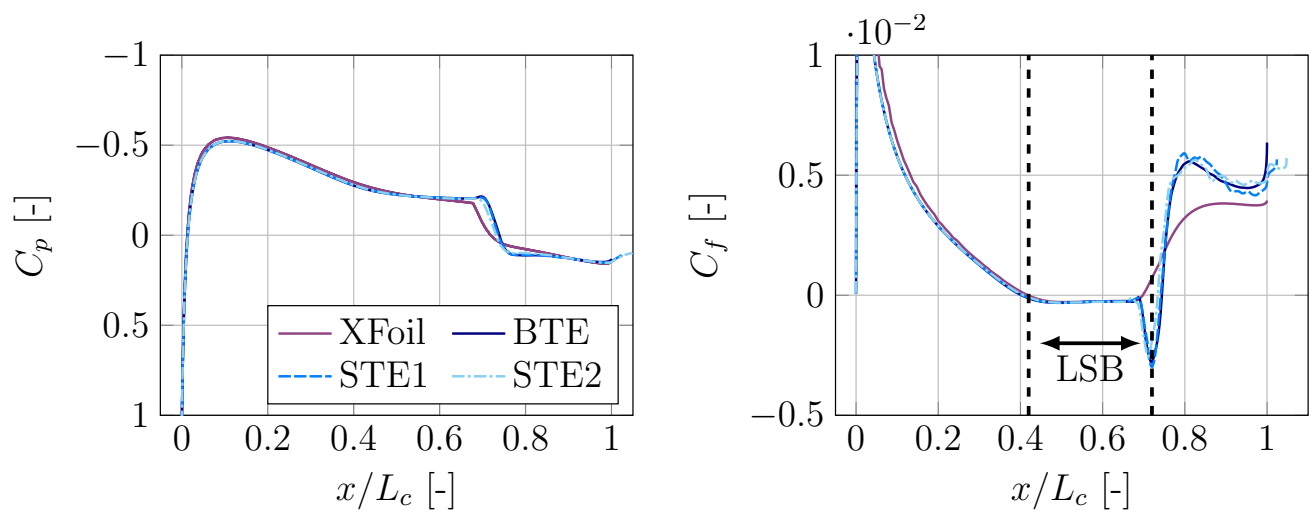

Figure 3. Time-averaged profiles of the pressure coefficient $C_{p}$ and skin friction coefficient $C_{f}$. The BTE profile is spanwise-averaged whereas the STE profiles are extracted from the mid-span cross-section. The laminar separation bubble (LSB) range is also depicted.

\subsection{Calculation of the acoustic field}

For the current study, an established approach originally proposed by Ffowcs-Williams and Hawkings (FW-H) is adopted to calculate the acoustic solutions in the far field. The 'Formulation 1A' derived by Farassat (2007) is implemented in this paper. Since the monopole and quadrupole contributions are irrelevant to the current study, the loading noise is solely considered and the following equation is used:

$$
\begin{aligned}
4 \pi p_{L}^{\prime}= & \int_{S}\left[\frac{\dot{p} \cos \theta}{a_{\infty} r\left(1-M_{r}\right)^{2}}+\frac{\hat{r}_{i} \dot{M}_{i} p \cos \theta}{a_{\infty} r\left(1-M_{r}\right)^{3}}\right]_{\mathrm{ret}} \mathrm{d} S \\
& +\int_{S}\left[\frac{p\left(\cos \theta-M_{i} n_{i}\right)}{r^{2}\left(1-M_{r}\right)^{2}}+\frac{\left(M_{r}-M_{\infty}^{2}\right) p \cos \theta}{r^{2}\left(1-M_{r}\right)^{3}}\right]_{\text {ret }} \mathrm{d} S
\end{aligned}
$$

where subscript $L$ designates the loading noise component; '.' is the time derivative; 'ret' stands for 'retarded time'; $M_{\infty}=|\mathbf{M}|$ is the Mach number; $\hat{\mathbf{r}}$ is the unit radiation vector between the source and the observer; $r$ is the source-observer distance; $\cos \theta=\mathbf{n} \cdot \hat{\mathbf{r}} ; M_{r}=$ $\mathbf{M} \cdot \hat{\mathbf{r}}$; and $S$ is the surface of integration. A numerical validation of this implementation can be found in Turner \& Kim (2020). A constant non-dimensionalised radius of 10 is taken for all the presented results and appropriate observer angles are specified later on. 
Moreover, for the range of frequencies exposed $\left(1<f L_{c} / a_{\infty}<25\right), f r / a_{\infty}>10$ so the observer truly lies in the far field.

\subsection{Definition of variables for statistical analysis}

Data processing and analysis are carried out upon the completion of each simulation. The main property required in this study is the power spectral density (PSD) function of pressure fluctuations on the aerofoil surface and at the far field observer location. The pressure fluctuations is defined as:

$$
p^{\prime}(\boldsymbol{x}, t)=p(\boldsymbol{x}, t)-\bar{p}(\boldsymbol{x}),
$$

where $\bar{p}(\boldsymbol{x})$ is the time-averaged pressure. Following Goldstein (1976), the one-sided PSD functions of the pressure fluctuations based on frequency are defined by:

$$
S_{p p}(\boldsymbol{x}, f)=\lim _{T \rightarrow \infty} \frac{P(\boldsymbol{x}, f, T) P^{*}(\boldsymbol{x}, f, T)}{T},
$$

where $P$ is an approximate Fourier transform of $p^{\prime}$ based on the following definition:

$$
P(\boldsymbol{x}, f, T)=\int_{-T}^{T} p^{\prime}(\boldsymbol{x}, t) e^{2 \pi \mathrm{i} f t} \mathrm{~d} t,
$$

and, '*' denotes a complex conjugate. In the above equations, $T$ represents the half length of the time signals used for the approximate Fourier transform. The present study also exhibits the wall pressure jump which is defined in the frequency domain by:

$$
\Delta P(\boldsymbol{x}, f, T)=\int_{-T}^{T} \Delta p^{\prime}(\boldsymbol{x}, t) e^{2 \pi \mathrm{i} f t} \mathrm{~d} t
$$

where $\Delta p^{\prime}=p_{u}^{\prime}-p_{l}^{\prime}$ is the difference between the pressure fluctuations on the upper and the lower sides of the aerofoil.

\section{Results and discussion}

The results of the current study are presented under three subsections. The first part shows how the acoustic feedback loop and the corresponding tonal noise are retrieved from the current LES data. It also depicts how the upstream travelling acoustic wave affects the laminar separation bubble. The second part discusses the tonal noise abatement in the presence of trailing edge serrations. The third part investigates the effect of the spanwise domain length.

\subsection{Acoustic feedback loop generating a tonal noise}

Figure $4 a$ shows some surface contour plots of the wall pressure fluctuations at five different frequencies, obtained from (2.6), for the BTE case. Only the second half of the chord is displayed for clarity. Figure $4 b$ shows spanwise-averaged PSDs of the wall pressure fluctuations measured at a few different streamwise locations. The first observation made from figure 4 is that the tonal peak at $f L_{c} / a_{\infty} \simeq 3.9$, is originated from the local area $\left(x / L_{c} \simeq 0.72\right)$ where the boundary-layer transition occurs. As indicated earlier, the tonal peak is generated by an acoustic feedback loop which has extensively been investigated by Jones \& Sandberg (2011). The present results are compared with the findings of Arbey \& Bataille (1983). First, it is necessary to estimate the convection velocity of the tonal component in the turbulent boundary layer. For this purpose, a wavenumber-frequency Fourier transform of the streamwise velocity fluctuation is 
(a)

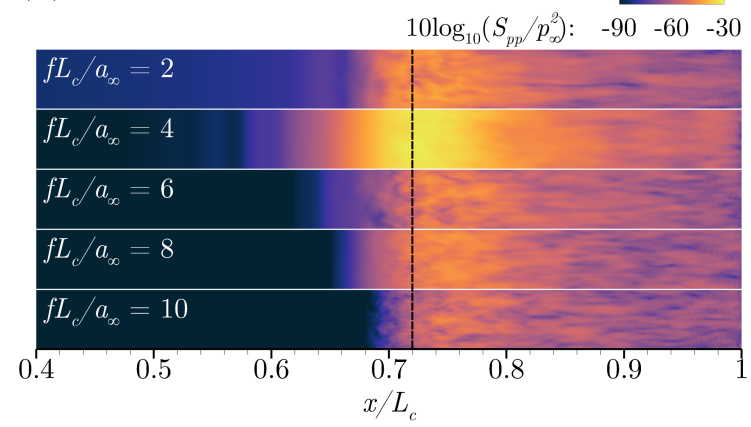

(b)

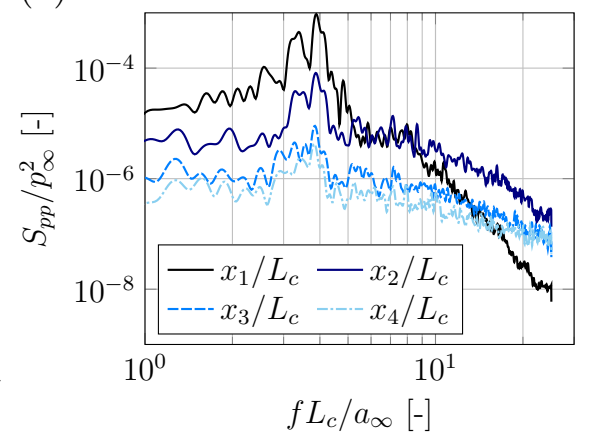

Figure 4. (a) Contour maps of the PSD of wall pressure fluctuations (WPF) on the suction side of the baseline aerofoil in log scale at five different frequencies, where the black dashed line indicates the transition location; and, $(b)$ spanwise-averaged PSDs of WPF on the suction side extracted at four different streamwise locations: $x_{1} / L_{c}=0.72, x_{2} / L_{c}=0.80, x_{3} / L_{c}=0.90$ and $x_{4} / L_{c}=0.99$.

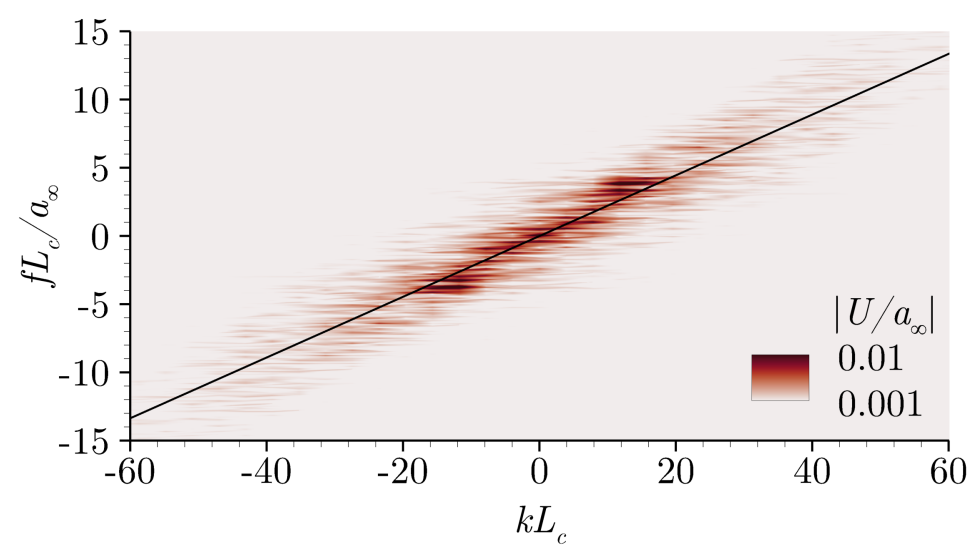

FIGURE 5. A wavenumber-frequency spectrum of the streamwise velocity fluctuation (the magnitude of the Fourier transform) extracted on a mid-span plane along a path-line displaced from the wall by about $20 \%$ of the boundary layer thickness (at the TE). Least square best fit line with a slope of 0.223 is also displayed.

computed on a mid-span plane along a path-line which extends from $x / L_{c}=0.8$ to 1.0 and displaced from the wall by about $20 \%$ of the boundary layer thickness (at the TE). The resulting contour map of the wavenumber-frequency Fourier transform (magnitude) is displayed in figure 5. Based on the data, a least square best fit is performed (as shown in the figure). The best fit gives an estimated convection speed of $U_{c} / a_{\infty}=0.223$ corresponding to $55.6 \%$ of the free-stream velocity. By using (1.1), a tonal frequency of $f L_{c} / a_{\infty}=3.81$ is predicted for $n=3$. This is within only a $2 \%$ deviation from the current LES result which confirms the presence of an acoustic feedback loop. In the far field noise spectra presented, the contribution of quadrupole sources is considered negligible for two reasons: 1) only the dipole term (surface integral) of the $\mathrm{FW}-\mathrm{H}$ calculation is used and 2) at the low free-stream Mach number of the current study, any footprint of the wake noise captured in the surface integral would be substantially weaker than the dipole contribution. Hence, the tonal noise calculated with the FW-H dipole formula may be solely attributed to Arbey \& Bataille's feedback model. 
A more in-depth investigation of the acoustic feedback loop is proposed in figure 6 . The solid grey contour lines show the upstream travelling acoustic waves (based on pressure fluctuations) at $f L_{c} / a_{\infty}=3.9$. Since the acoustic waves are masked by the turbulence in the near field, the authors have extrapolated them (dashed grey) with the following function:

$$
\zeta(\boldsymbol{x}, t)=\sin \left[2 \pi f_{0}\left(t-\frac{r(\boldsymbol{x})}{u_{a c}}\right)\right]
$$

where $f_{0}$ is the tonal frequency, $r(\boldsymbol{x})$ is the distance between the TE and the point $\boldsymbol{x}, u_{a c}$ is the acoustic velocity which depends on the flow speed. The vertical cross-stream velocity time derivative fluctuation $\mathrm{d} v / \mathrm{d} t$ emphasises the size variation of the laminar separation bubble canopy illustrated by the pink contour line. The quantity $\mathrm{d} v / \mathrm{d} t$ is filtered at the feedback frequency $f L_{c} / a_{\infty}=3.9$. The filtering is applied by a narrowband window as follows:

$$
\begin{gathered}
\widetilde{\mathrm{d} v}(\boldsymbol{x}, t)=\mathcal{F}^{-1}\left\{W(f) \mathcal{F}\left\{\frac{\mathrm{d} v}{\mathrm{~d} t}(\boldsymbol{x}, t)\right\}\right\}, \\
W(f)=\sum_{m=0}^{1} \exp \left[-\alpha\left(f+(-1)^{m} f_{0}\right)^{2}\right]
\end{gathered}
$$

where $\mathcal{F}$ and $\mathcal{F}^{-1}$ represent the Fourier and inverse Fourier transforms, respectively; ' $\simeq$, denotes a filtered variable; $\alpha$ is a damping parameter $(\alpha=5)$ and $f_{0}$ is the frequency around which the signal is to be filtered. It can be seen from the plots that a detached LSB is generated when the expansion wave (blue arrow) hits the main LSB. Moreover, $\mathrm{d} v / \mathrm{d} t$ shows in figure 6 an increased magnitude on the edge of the LSB as time marches emphasising the fact that the LSB is excited by the incoming acoustic expansion wave and its tail broadens as the secondary LSB detaches. The incoming acoustic wave front and the LSB canopy are structures from two different scales, therefore a close-up of the LSB canopy is proposed in figure 7 along with a velocity vector field visualisation to picture the LSB detachment more accurately. Figure 8 displays the time evolution of the bubble canopy. The time frame is close to the feedback period and the periodic detachment of the secondary LSB is well illustrated by the white dashed lines.

\subsection{Influence of serrated trailing edges on the acoustic feedback loop}

Instantaneous plots of the generated sound waves are illustrated in figure 9 based on the divergence of velocity $\partial\left(u_{j} / a_{\infty}\right) / \partial\left(x_{j} / L_{c}\right)$. It should be noted here that the mesh resolution is maintained sufficiently fine for the high-frequency components up to about half a chord length away from the aerofoil surface. Figure 9 compares the BTE, STE1 and STE2 cases. It is noticeable from the figure that the overall intensity of the sound waves becomes weaker as a sharper STE is used, compared to the BTE case.

More details of the noise reduction due to the STEs are further investigated in figure 10 where the loading noise from (2.4) is explored. The far field sound pressure fluctuations spectra in figure $10 a$ clearly show the tonal noise component at $f L_{c} / a_{\infty}=3.9$ in the BTE case, which becomes significantly reduced when the STEs are used. Figure $10 b$ presents the relative change of the spectra (due to the use of the STEs) in a decibel scale. It is observed that a $10 \mathrm{~dB}$ reduction of the tonal noise is obtained in the STE2 case. Meanwhile, the noise increase observed at lower frequencies might be due to the usage of a reasonably small spanwise domain size - as figure 17 suggests that a longer spanwise domain size tend to lower the peak and shift it towards even lower frequencies - as well as a time signal not sufficiently long to accurately address low frequency events. 

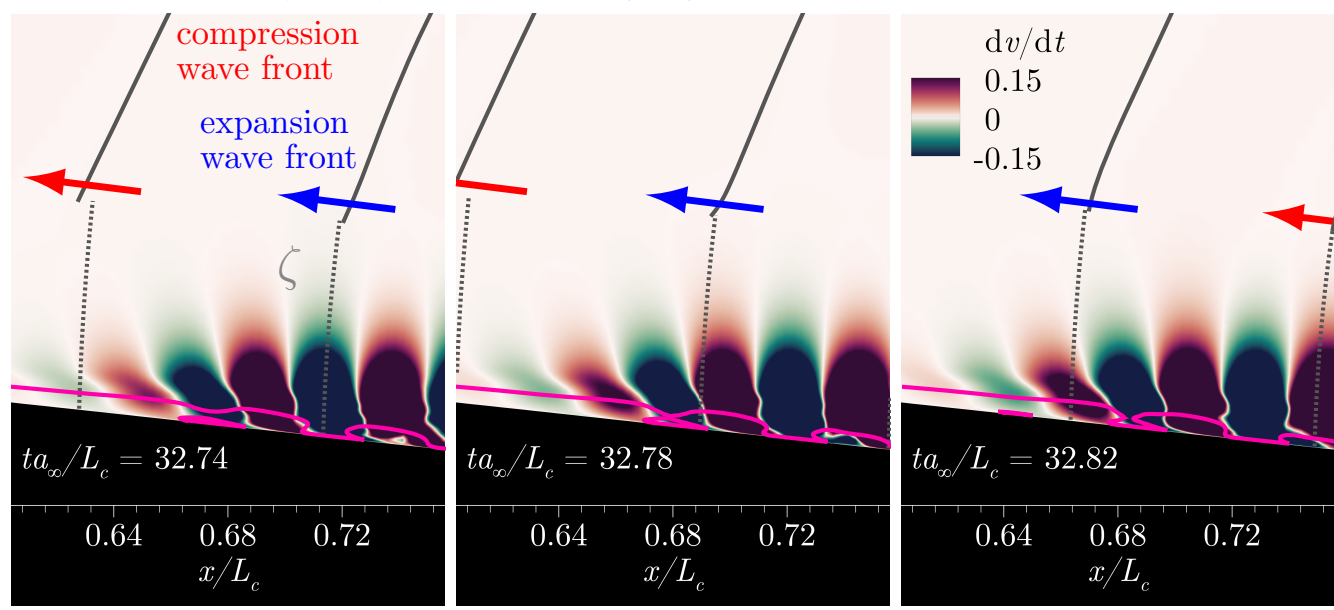

FiguRE 6. Visualised acoustic feedback loop in a mid-span plane showing flood of filtered vertical cross-stream velocity time derivative fluctuation $\mathrm{d} v / \mathrm{d} t$ at $f L_{c} / a_{\infty}=3.9$, contour lines of acoustic wave front (solid grey), extrapolated acoustic wave front in the near field (dashed grey) and the laminar separation bubble canopy (solid pink).
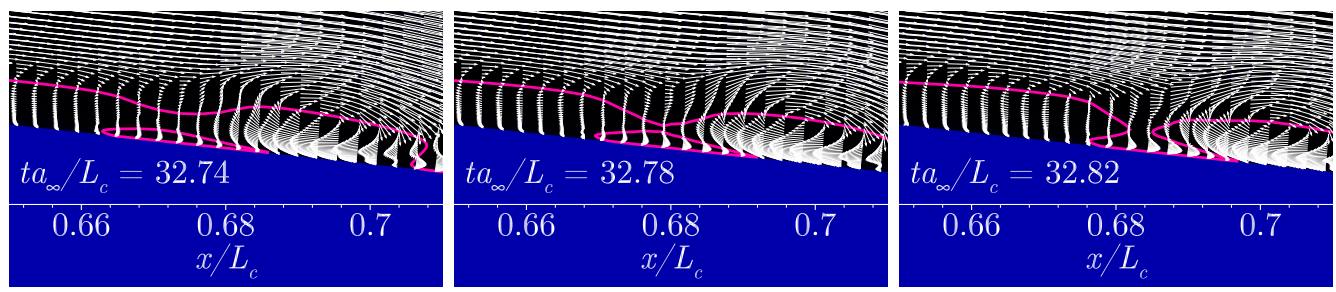

Figure 7. Close-up on the velocity vector field near the LSB detachment. Snapshots are synchronised with the ones in figure 6 .

Although the magnitudes are captured more accurately by the narrowband spectra on figure 10, figure 11 gives a better insight in the spectrum trend by showing one-thirdoctave band $(a)$ individual PSDs and $(b)$ SPL differences. At higher frequencies, it is relatively clear that STE1 does not produce any broadband noise reduction whereas STE2 performs better with an attenuation of $4 \mathrm{~dB}$ compared to BTE at $f L_{c} / a_{\infty}=20$. This result is in agreement with the past investigations conducted by Gruber et al. (2011) on serrations. They demonstrated that significant noise reduction occur above a critical serration aspect ratio of 1.0 (corresponding to STE2 in the present study).

Figure 12 shows the acoustic source pressure distribution at the tonal frequency quantified by a Fourier transform of the wall pressure jump between the upper and lower surfaces of the aerofoil (see equation (2.8)). In this paper, the proposed mechanisms of the tonal noise reduction due to the STEs are twofold: 1) decreased magnitude of the acoustic source pressure in the transitional region (figure 12a) and 2) incoherent phase distribution of the acoustic source downstream of the transitional region (figure $12 b)$. A reduced intensity of the acoustic source pressure at the transition when the serrated geometries are used results in a reduced intensity of the acoustic source at the $\mathrm{TE}$ and consequently means that the acoustic excitation of the transitional separation bubble upstream becomes less effective. The reduced intensity of the acoustic source at the transition in the serrated cases (figure 12a) could be attributed to the laminar separation bubble behaviour in this region. As depicted in figure 8, a secondary LSB detaches from the main LSB with a clear periodic pattern in the BTE case whereas in 


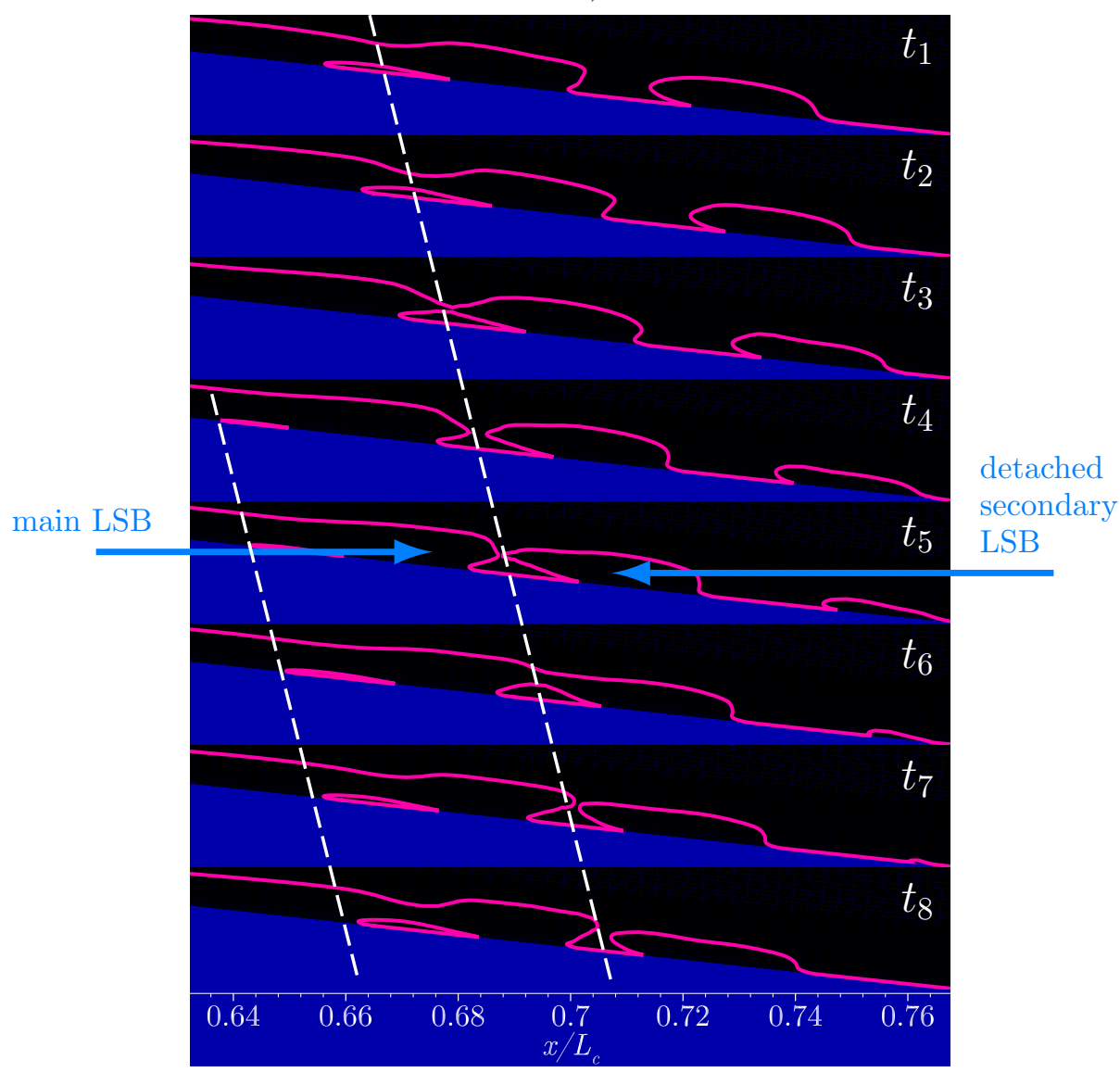

FiguRE 8. Time evolution of the laminar separation bubble over a period $\left(t_{8}-t_{1}\right) a_{\infty} / L_{c}=0.28$ in the BTE case. Frames are extracted with a time step of $(\Delta t) a_{\infty} / L_{c}=0.04$. The white dashed lines depict the periodic detachment of the secondary LSB.
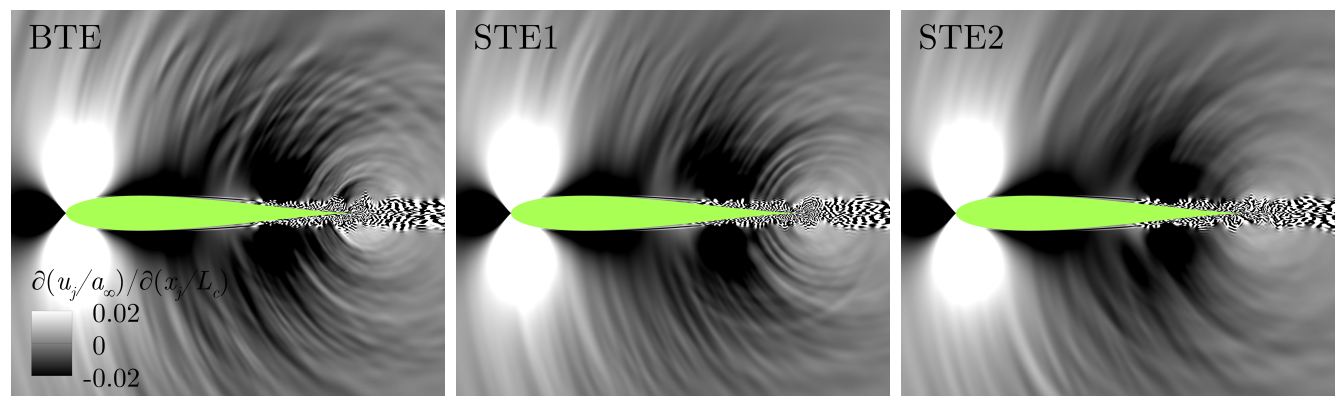

Figure 9 . Contour map of the divergence of velocity $\partial\left(u_{j} / a_{\infty}\right) / \partial\left(x_{j} / L_{c}\right)$ on a mid-span plane comparing the BTE, STE1 and STE2 cases.

the STE2 case (figure 13), the secondary LSB re-merges with the main LSB and weaker recirculation is produced. The authors theorise that the merging of LSBs inhibits the secondary LSB detachment and that the absence of LSB detachment may explain the reduced amplitude of acoustic source pressure in the transitional region at the tonal frequency. Figure 14 provides the profiles of the source phase variation (at the tonal frequency) as a function of the spanwise coordinate, obtained at the transition location 

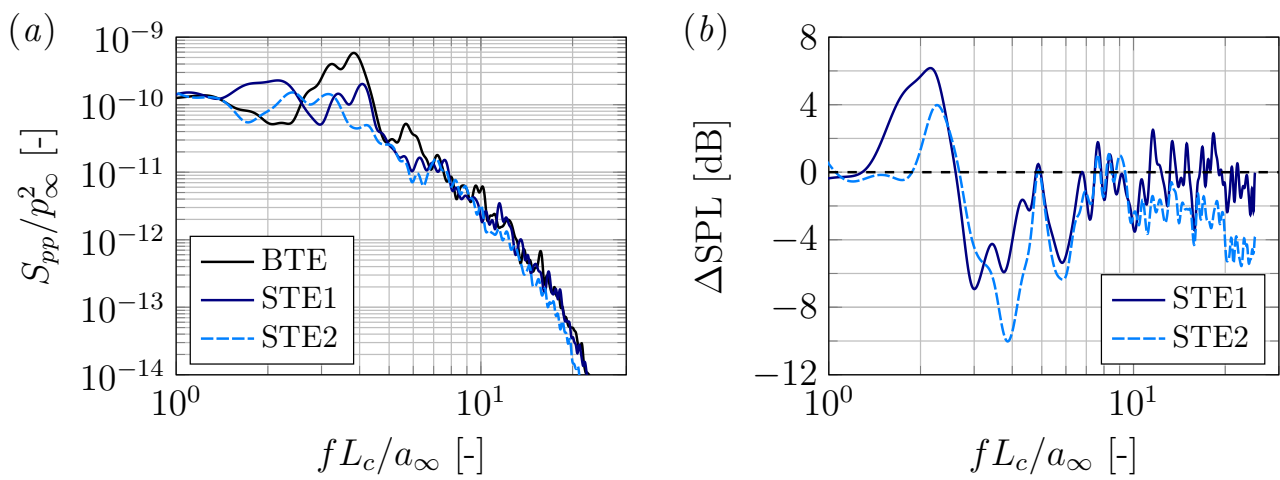

Figure 10. Power spectral density of far field sound pressure fluctuations (loading noise from (2.4)) over a narrow circular arc $80^{\circ} \leqslant \theta \leqslant 100^{\circ}$ of a radius of $r / L_{c}=10$ centered at the TE of the baseline aerofoil $\left(x / L_{c}, y / L_{c}, z / L_{c}\right)=(1.0,0,0):(a)$ the individual PSDs and $(b)$ the relative difference of the $\mathrm{STE}$ cases to the baseline in decibels.
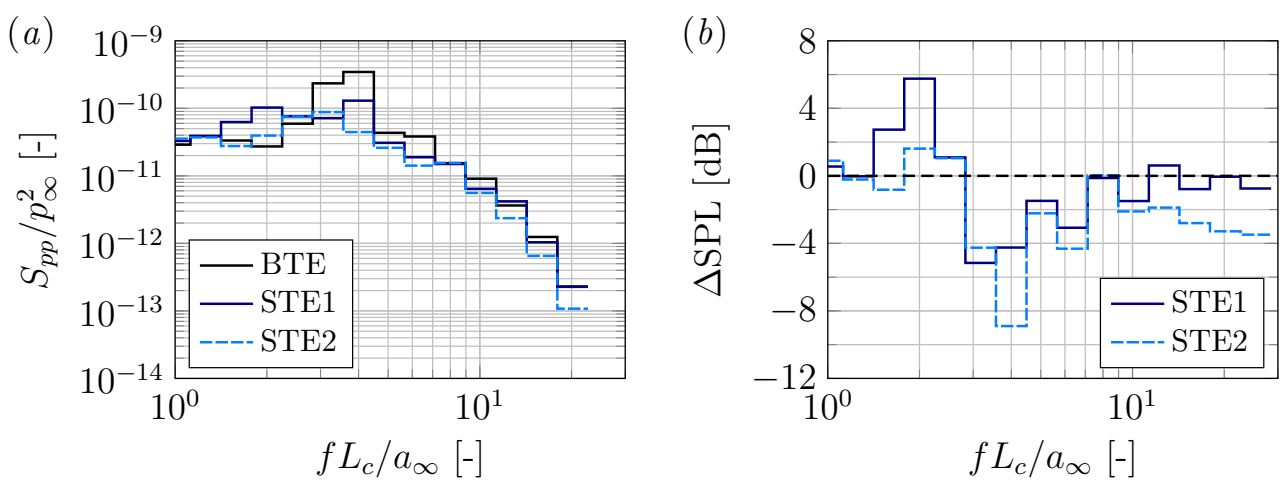

FiguRE 11. One-third-octave band power spectral density of far field sound pressure fluctuations (loading noise from (2.4)) over a narrow circular arc $80^{\circ} \leqslant \theta \leqslant 100^{\circ}$ of a radius of $r / L_{c}=10$ centered at the TE of the baseline aerofoil $\left(x / L_{c}, y / L_{c}, z / L_{c}\right)=(1.0,0,0):(a)$ the individual PSDs and $(b)$ the relative difference of the STE cases to the baseline in decibels.

and at the TE. The reference phase is set to the spanwise-averaged value at the transition location, for each of the three cases. The graphs show that there is a significant phase variation as much as $180^{\circ}$ in the STE cases whereas the baseline case maintains a highly uniform distribution. This variation is likely to produce some destructive interference and supports the second mechanism proposed in this paper. There exist similar phase interference effects discussed for TE broadband noise (Avallone et al. 2018) and leading edge interaction noise (Turner \& Kim 2019).

\subsection{Impact of the spanwise domain length}

For completeness of the present study, a span length investigation is also reported below. On top of the three cases BTE, STE1 and STE2 performed with 5\% span (one serration resolved), BTE-ext. and STE2-ext. cases have been run using an extended $10 \%$ span (two serrations resolved). Figures 15 and 16 compare the $C_{p}$ and $C_{f}$ profiles obtained with the two different spanwise domain sizes for BTE and STE2 cases. In both cases, the results are very close. Figures 17 and 18 display the FW-H comparison. The integration uses juxtaposed aerofoils. While BTE and STE2 were integrated over $0.05 L_{c} \times 401=20.05 L_{c}$ total span length, BTE-ext. and STE2-ext. were integrated 
$(a)$

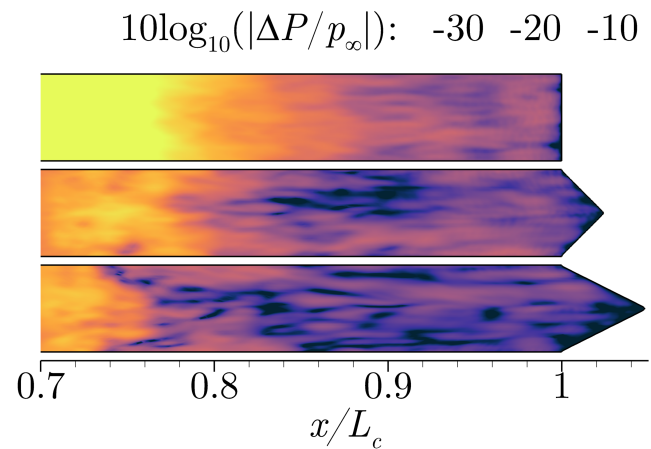

$(b)$

$$
\cos (\phi): \quad-1 \quad 0 \quad 1
$$

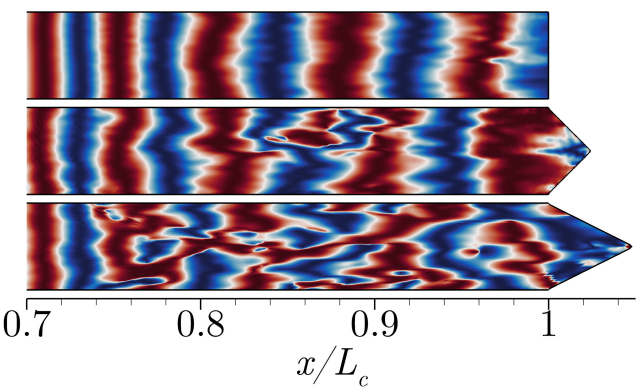

FiguRE 12. Surface contour maps of the Fourier transform of the wall pressure jump at the tonal frequency $f L_{c} / a_{\infty}=3.9$ : $(a)$ the magnitude in log scale and $(b)$ the phase distribution. The reference phase $\phi_{\text {ref }}$ is the spanwise averaged value at the transition location $\left(x / L_{c}=0.72\right)$.

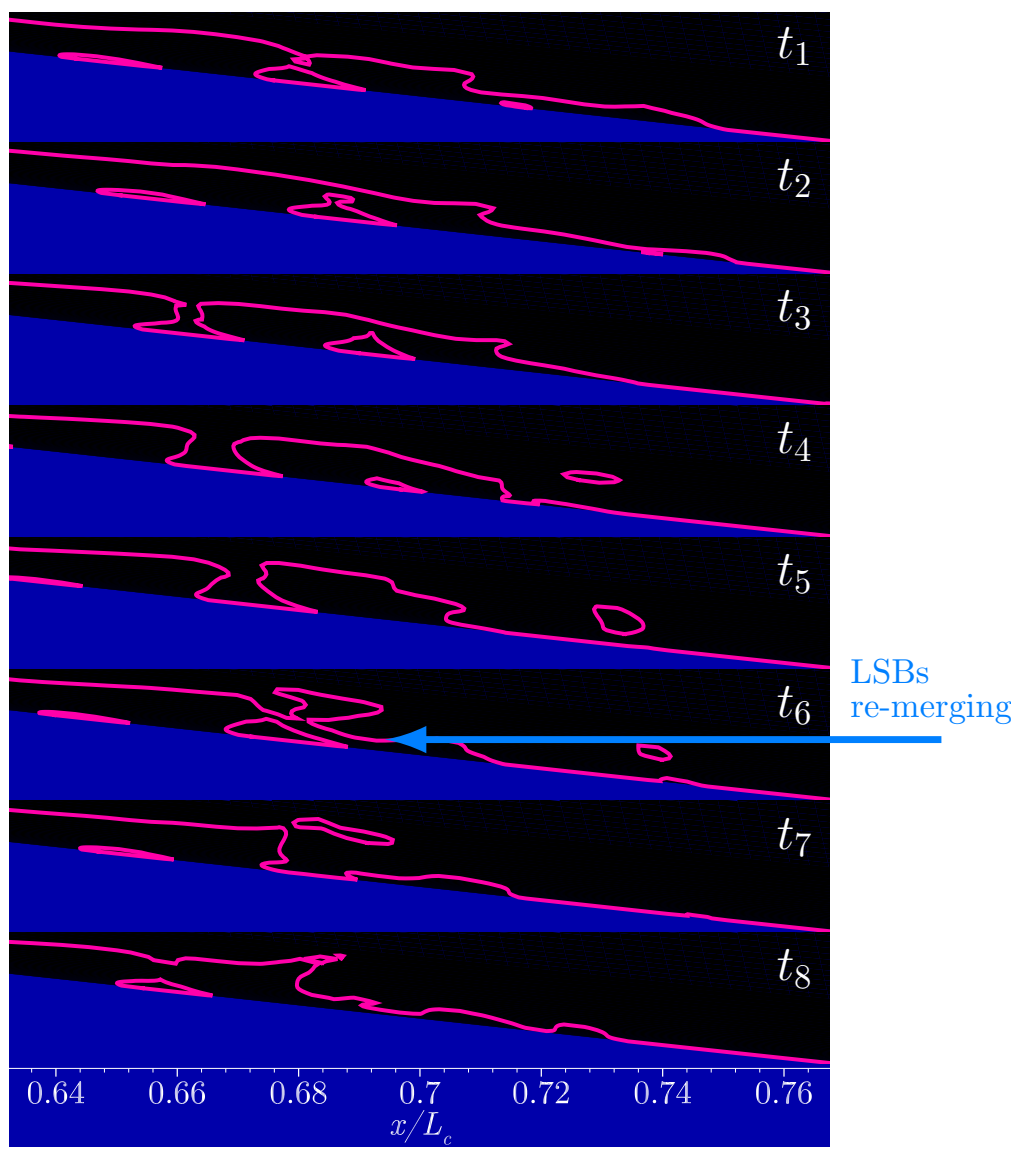

FiguRE 13. Time evolution of the laminar separation bubble over a period $\left(t_{8}-t_{1}\right) a_{\infty} / L_{c}=0.28$ in the STE2 case. Frames are extracted with a time step of $(\Delta t) a_{\infty} / L_{c}=0.04$. 
$(a)$

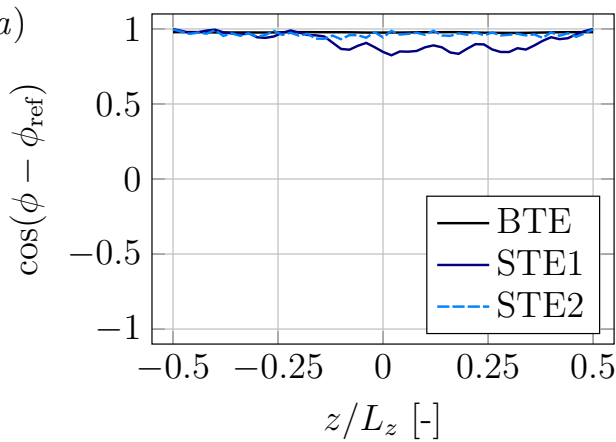

$(b)$

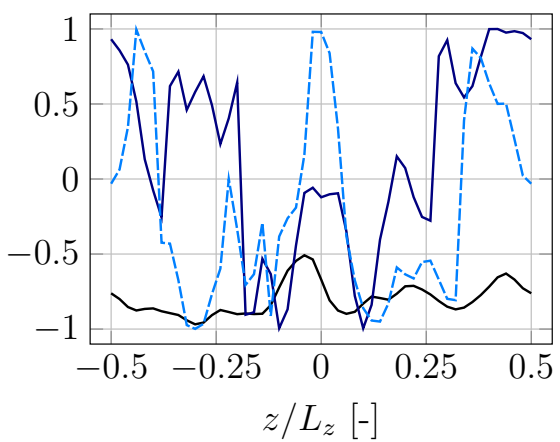

Figure 14. Spanwise variation of the phase of the wall pressure fluctuations taken at the tonal frequency $f L_{c} / a_{\infty}=3.9$ : $(a)$ at the transition location and (b) along the TE.
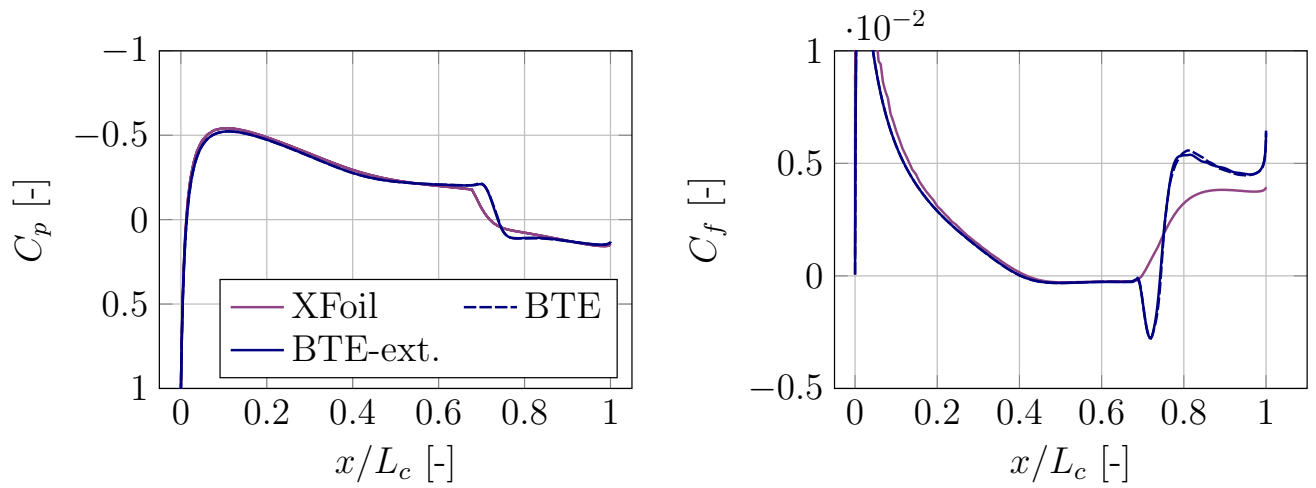

FiguRE 15. Time-averaged profiles of the pressure coefficient $C_{p}$ and skin friction coefficient $C_{f}$. The BTE profiles are spanwise-averaged.

over $0.1 L_{c} \times 201=20.10 L_{c}$ total span length making the total span length comparable. Furthermore, the FW-H calculation is already converged when the span is repeated to $20 L_{c}$. BTE and BTE-ext. give a very similar far field sound and it is clear that STE2-ext. provides a clearer trend of noise reduction compared to STE2 at the tonal frequency. The broadband component remains the same at higher frequencies. The exploration of the acoustic source (wall pressure jump) in figure $19 a$ explains the above observation: in the $10 \%$ span case, the magnitude of acoustic source pressure in the transitional region is slightly lower than in the $5 \%$ span case. Furthermore, the phase distribution shows more variation along the TE (figure 19b). This phenomenon is emphasised in figure 20 where the phase along the serrated trailing edge is compared between STE2 and STE2-ext. cases. In the $10 \%$ span case, greater variations of the phase are detected. Hence, both mechanisms previously inferred are consistent enough to explain why the extended span case produces a clearer trend of sound attenuation.

\section{Conclusions}

This paper has investigated how the aerofoil tonal noise could be reduced due to a STE, based on a large-eddy simulation of a Joukowski aerofoil with a $12 \%$ thickness at $R e_{\infty}=$ $250000, M_{\infty}=0.4$ and zero incidence angle. The current simulation properly captured the well-known acoustic feedback loop and the consequent tonal noise generation. The interaction of the upstream travelling acoustic wave with the laminar separation bubble 

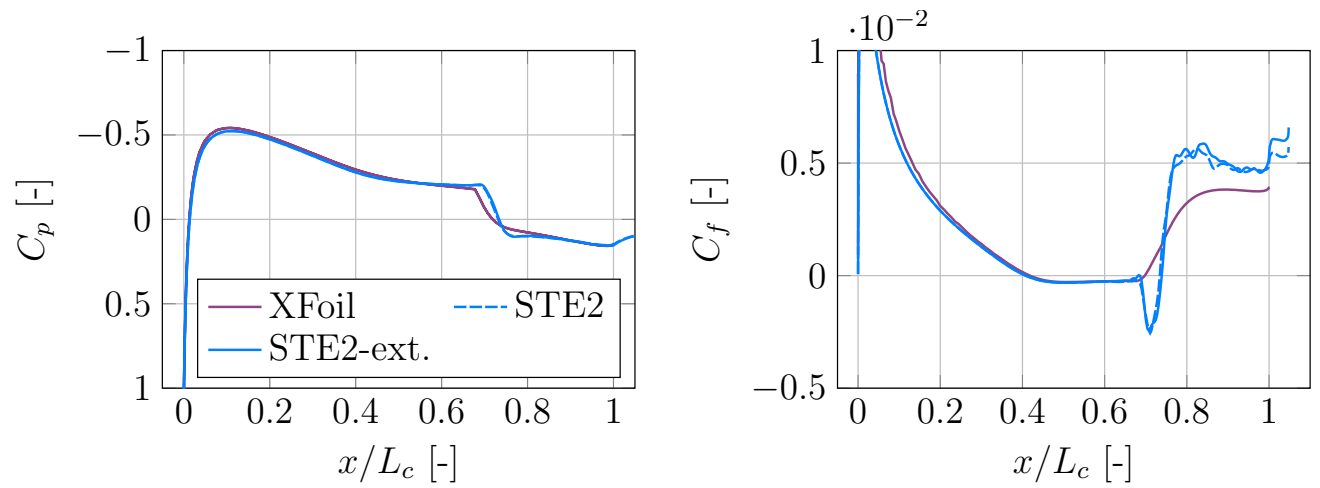

Figure 16. Time-averaged profiles of the pressure coefficient $C_{p}$ and skin friction coefficient $C_{f}$. The STE profiles are extracted at the tip of the serrations.
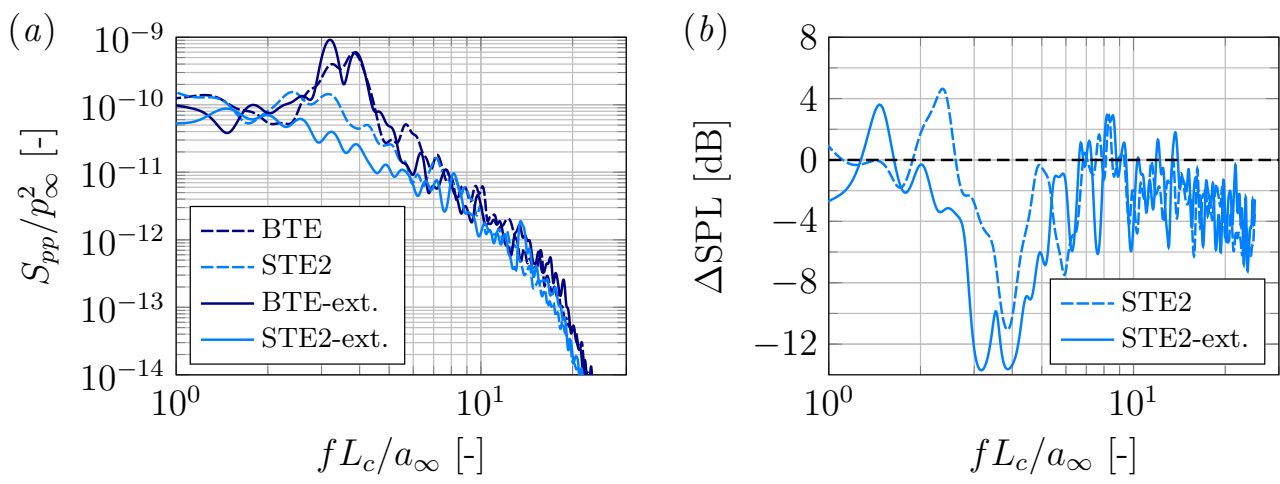

FiguRE 17. Power spectral density of far field sound pressure fluctuations (loading noise from (2.4)) over a narrow circular arc $80^{\circ} \leqslant \theta \leqslant 100^{\circ}$ of a radius of $r / L_{c}=10$ centered at the TE of the baseline aerofoil $\left(x / L_{c}, y / L_{c}, z / L_{c}\right)=(1.0,0,0)$ comparing two span lengths: $(a)$ the individual PSDs and $(b)$ the relative difference of the STE2 cases to the baseline in decibels.
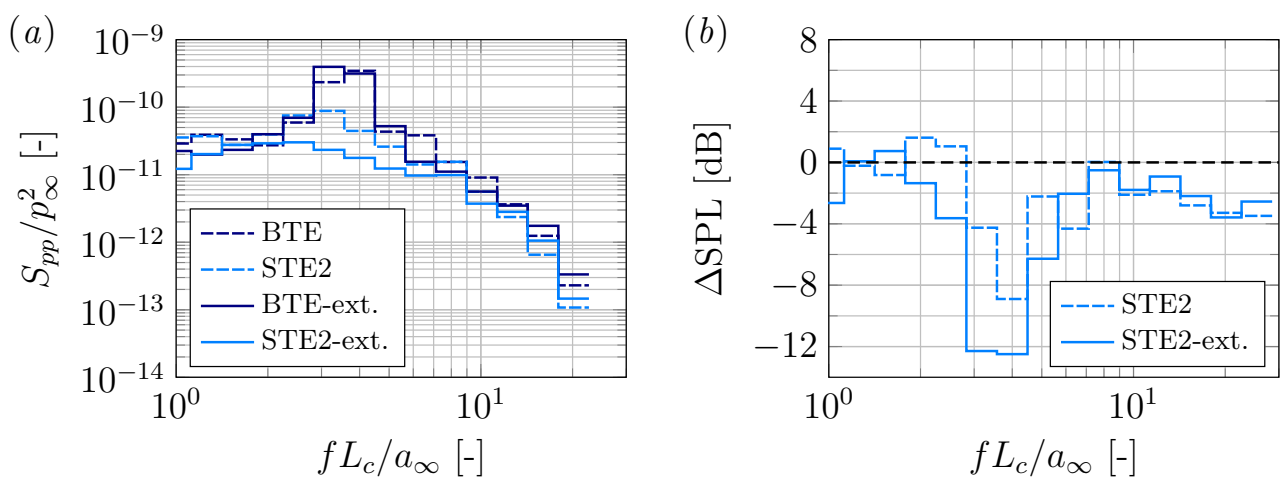

FiguRE 18. One-third-octave band power spectral density of far field sound pressure fluctuations (loading noise from (2.4)) over a narrow circular arc $80^{\circ} \leqslant \theta \leqslant 100^{\circ}$ of a radius of $r / L_{c}=10$ centered at the TE of the baseline aerofoil $\left(x / L_{c}, y / L_{c}, z / L_{c}\right)=(1.0,0,0)$ comparing two span lengths: $(a)$ the individual PSDs and $(b)$ the relative difference of the STE2 cases to the baseline in decibels. 
(a)

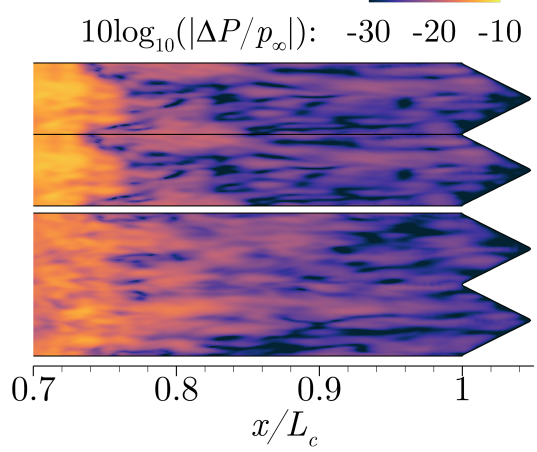

(b)

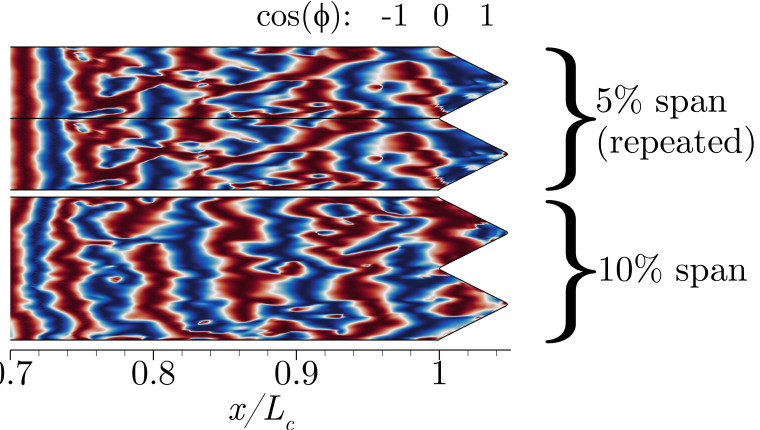

Figure 19. Comparison of $5 \%$ span (top) and $10 \%$ span (bottom) for STE2 case with surface contour maps of the Fourier transform of the wall pressure jump at the tonal frequency $f L_{c} / a_{\infty}=3.9$ : $(a)$ the magnitude in $\log$ scale and $(b)$ the phase distribution.

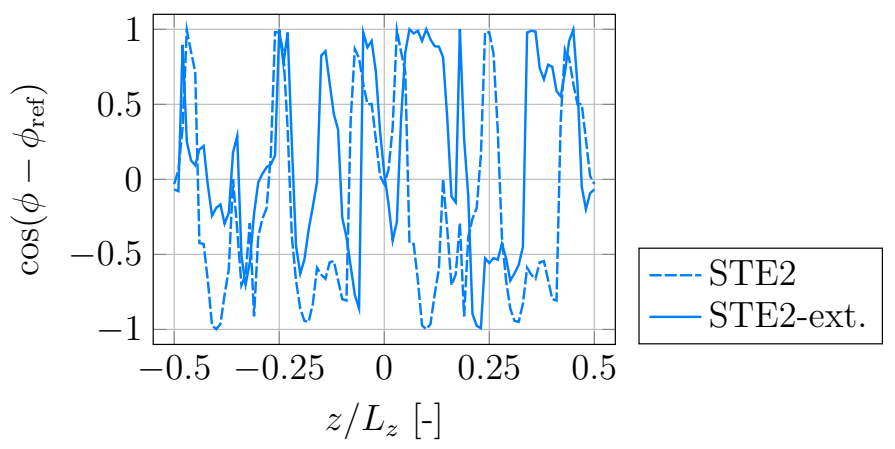

Figure 20. Comparison of the spanwise variation of the phase of the wall pressure fluctuations taken at the tonal frequency $f L_{c} / a_{\infty}=3.9$ along the TE between STE2 and STE2-ext.. $L_{z}=0.1 L_{c}$ and the distribution for STE2 is repeated two times.

was studied in detail. It has thus been shown that the incoming acoustic expansion wave directly excites the main LSB giving birth to detached secondary LSBs at the tonal frequency. Also, the significant reduction of the tonal noise due to the STEs which Chong \& Joseph (2013) reported, is validated by the current LES results. It has been demonstrated that using a sawtooth STE with a length of $5 \%$ of the chord and an aspect ratio of 1.0 achieved a $10 \mathrm{~dB}$ noise reduction of the tonal noise, in the present aerofoil geometry and flow condition used. A secondary investigation reported a comparison of two spanwise domain sizes (5\% and $10 \%$ chord respectively) and it was found that the extended span case gives a clearer trend of noise reduction at the tonal frequency. Two major mechanisms of the tonal noise reduction were suggested in this paper. The first was the reduced intensity of acoustic source pressure (or wall pressure jump) in the transitional region in the presence of STE which directly weakens the scattering at the TE. The second was the early breakdown of the spanwise coherence in the acoustic source which is likely to create destructive interference in the acoustic feedback process. The two inferred mechanisms are also comprehensible in explaining the clearer trend of noise abatement shown by the extended span case. 


\section{Acknowledgements}

We would like to acknowledge the support of VESTAS for this work, with special thanks to Dr Ronan Serré, Mr Diogo Samora Cerqueira and Mr Tomas Vronsky. We also would like to thank EPSRC for the computational time made available on the UK supercomputing facility ARCHER via the UK Turbulence Consortium (EP/R029326/1), and the local IRIDIS-4 at the University of Southampton. All data supporting this study are openly available from the University of Southampton repository at doi:10.5258/SOTON/XXXXX. The authors report no conflict of interest.

\section{REFERENCES}

Arbey, H. \& Batallle, J. 1983 Noise generated by airfoil profiles placed in a uniform laminar flow. J. Fluid Mech. 134, 33-47.

Arcondoulis, E. J. G., Doolan, C. J., Zander, A. C. \& Brooks, L. A. 2013 An experimental investigation of airfoil tonal noise caused by an acoustic feedback loop. In Annu. Conf. Aus. Acoust. Soc. 2013, pp. 23-30.

Avallone, F., van der Velden, W. C. P., Ragni, D. \& Casalino, D. 2018 Noise reduction mechanisms of sawtooth and combed-sawtooth trailing-edge serrations. J. Fluid Mech. 848, 560-591.

Brooks, T. F., Pope, D. S. \& Marcolini, M. A. 1989 Airfoil self-noise and prediction. Tech. Rep.. NASA.

Chong, T. P. \& Joseph, P. F. 2013 An experimental study of airfoil instability tonal noise with trailing edge serrations. J. Sound Vib. 332 (24), 6335-6358.

Chong, T. P., Joseph, P. F. \& Kingan, M. J. 2013 An investigation of airfoil tonal noise at different Reynolds numbers and angles of attack. Appl. Acoust. 74, 38-48.

Desquesnes, G., Terracol, M. \& Sagaut, P. 2007 Numerical investigation of the tone noise mechanism over laminar airfoils. J. Fluid Mech. 591, 155-182.

FARAssat, F. 2007 Derivation of Formulations 1 and 1A of Farassat. Tech. Rep. March. NASA.

FinK, M. R. 1975 Prediction of airfoil tone frequencies. J. Aircraft 12 (2), 118-120.

Garmann, D. J., Visbal, M. R. \& Orkwis, P. D. 2013 Comparative study of implicit and subgrid-scale model large-eddy simulation techniques for low-Reynolds number airfoil applications. Int. J. Numer. Meth. Fl. 71 (12), 1546-1565.

Georgiadis, N. J., Rizzetta, D. P. \& Fureby, C. 2010 Large-Eddy Simulation: Current Capabilities, Recommended Practices, and Future Research. AIAA J. 48 (8), 1772-1784.

Goldstein, M. E. 1976 Aeroacoustics. McGraw-Hill.

Gruber, M., Joseph, P. F. \& Chong, T. P. 2011 On the mechanisms of serrated airfoil trailing edge noise reduction. In 17th AIAA/CEAS Aeroacoust. Conf., pp. 1-23.

Jones, L. E. \& SANDberg, R. D. 2011 Numerical analysis of tonal airfoil self-noise and acoustic feedback-loops. J. Sound Vib. 330 (25), 6137-6152.

KIM, J. W. 2007 Optimised boundary compact finite difference schemes for computational aeroacoustics. J. Comput. Phys. 225, 995-1019.

KIM, J. W. 2010 High-order compact filters with variable cut-off wavenumber and stable boundary treatment. Comput. Fluids 39, 1168-1182.

KIM, J. W. 2013 Quasi-disjoint pentadiagonal matrix systems for the parallelization of compact finite-difference schemes and filters. J. Comput. Phys. 241, 168-194.

Kim, J. W., LAU, A. S. H. \& Sandham, N. D. $2010 a$ CAA boundary conditions for airfoil noise due to high-frequency gusts. Procedia Eng. 6, 244-253.

Kim, J. W., Lau, A. S. H. \& Sandham, N. D. $2010 b$ Proposed Boundary Conditions for Gust-Airfoil Interaction Noise. AIAA J. 48 (11), 2705-2710.

KIM, J. W. \& LEE, D. J. 2000 Generalized characteristic boundary conditions for computational aeroacoustics. AIAA J. 38 (11), 2040-2049.

Longhouse, R. E. 1977 Vortex shedding noise of low tip speed, axial flow fans. J. Sound Vib. 53 (1), 25-46.

McAlpine, A., NAsh, E. C. \& Lowson, M. V. 1999 On the generation of discrete frequency tones by the flow around an aerofoil. J. Sound Vib. 222 (5), 753-779. 
Nash, E. C., Lowson, M. V. \& McAlpine, A. 1999 Boundary-layer instability noise on aerofoils. J. Fluid Mech. 382, 27-61.

Nguyen, L. D., Golubev, V. V., Mankbadi, R. R., Yakhina, G., Roger, M., Pasiliao, C. L. \& Visbal, M. R. 2017 On ladder-type structure of acoustic tones radiated by transitional airfoils. In 23rd AIAA/CEAS Aeroacoust. Conf., pp. 1-14.

Padois, T., Laffay, P., Idier, A. \& Moreau, S. 2016 Tonal noise of a controlled-diffusion airfoil at low angle of attack and Reynolds number. J. Acoust. Soc. Am. 140 (1), 113-118.

Paterson, R. W., Vogt, P. G., Fink, M. R. \& Munch, C. L. 1973 Vortex noise of isolated airfoils. J. Aircraft 10 (5), 296-302.

Pröbsting, S., Scarano, F. \& Morris, S. C. 2015 Regimes of tonal noise on an airfoil at moderate Reynolds number. J. Fluid Mech. 780, 407-438.

Pröbsting, S., Serpieri, J. \& Scarano, F. 2014 Experimental investigation of aerofoil tonal noise generation. J. Fluid Mech. 747 (2), 656-687.

Sanjose, M., Jaiswal, P., Moreau, S., Towne, A., Lele, S. K. \& Mann, A. 2017 Laminar boundary layer instability noise. In 23rd AIAA/CEAS Aeroacoust. Conf., pp. 1-13.

TAm, C. K. W. 1974 Discrete tones of isolated airfoils. J. Acoust. Soc. Am. 55 (6), 1173-1177.

TAM, C. K. W. \& Ju, H. 2012 Aerofoil tones at moderate Reynolds number. J. Fluid Mech. 690, $536-570$.

Turner, J. M. \& Kim, J. W. 2019 On the universal trends in the noise reduction due to wavy leading edges in aerofoil-vortex interaction. J. Fluid Mech. 871, 186-211.

Turner, J. M. \& KIM, J. W. 2020 Effect of spanwise domain size on direct numerical simulations of airfoil noise during flow separation and stall. Physics of Fluids 32 (6), $1-15$.

White, F. M. 1991 Viscous fluid flows. McGraw-Hill. 